\title{
Abkehr von der Depressionspraxis
}

Besprechung des Urteils des Bundesgerichts 8C_841/2016

vom 30. November 2017 (zur Publikation vorgesehen)

Sabine Steiger-Sackmann *

Mit seinem Urteil vom 30. November 2017 kehrt das Bundesgericht von seiner Praxis ab, bei Versicherten mit leicht- bis mittelgradigen Depressionen zusätzlich zu den Leistungsvoraussetzungen für eine IV-Rente zu verlangen, dass eine Therapieresistenz vorliegt. Diese Änderung der Rechtsprechung ist zu begrüssen, wenn auch einzelne Ausführungen der Urteilsbegründung Anlass zu kritischen Einwänden geben.

Zitiervorschlag: Sabine Steiger-Sackmann, Abkehr von der Depressionspraxis, in: sui-generis 2017, S. 332

URL: $\quad$ sui-generis.ch/54

DOI: $\quad$ https://doi.org/10.21257/sg.54

* RA Dr. iur. Sabine Steiger-Sackmann, Dozentin für Arbeits- und Sozialversicherungsrecht, Zentrum für Sozialrecht, Zürcher Hochschule für Angewandte Wissenschaften. 


\section{Zusammenfassung des Urteils}

1 Die beiden sozialrechtlichen Abteilungen des Bundesgerichtes ändern mit ihrem zur Publikation vorgesehenen Entscheid eine Praxis, welche sich im Verlauf der letzten Jahre (ohne einen Leitentscheid) in die bundesgerichtliche Rechtsprechung eingeschlichen hatte. Danach erhielten Versicherte mit leicht- bis mittelgradigen Depressionen prinzipiell keine Invalidenrente, ausser die Krankheit hat sich als therapieresistent erwiesen.

2 Mit der Praxisänderung verabschiedet sich das Bundesgericht nun von dieser starren Beweisregel und findet zurück zur einzelfallgerechten Beweiswürdigung. Beweisthema ist nicht mehr länger die Frage, ob die zur Verfügung stehenden medizinischen Therapien ausgeschöpft sind, sondern welche Auswirkungen die ärztlich diagnostizierte Krankheit auf die Erwerbsmöglichkeiten hat unter Berücksichtigung der Schadenminderungspflicht bzw. Kooperation der versicherten Person bei therapeutischen Massnahmen.

Künftig soll bei sämtlichen psychischen Erkrankungen das strukturierte Beweisverfahren durchgeführt werden, welches mit BGE $141 \mathrm{~V} 281$ für somatoforme Schmerzstörungen und vergleichbare psychosomatischen Leiden etabliert wurde (so auch das gleichentags gefällte Urteil 8C_130/2017). In diesem Beweisverfahren werden in einer Gesamtbetrachtung das tatsächlich erreichbare Leistungsvermögen anhand von verschiedenen Indikatoren ermittelt und in diesem Kontext unter anderem der Schweregrad der medizinischen Befunde und die Inanspruchnahme und Wirkung einer Therapie berücksichtigt (E. 4.5.2).
4 Aus Gründen der Verhältnismässigkeit könne aber auf dieses strukturierte Beweisverfahren verzichtet werden, wenn dafür kein Bedarf bestehe, weil es nicht nötig oder nicht geeignet sei. Dies sei der Fall, wenn bei leichtgradigen Depressionen ohne Komorbidität nach fachärztlicher Beurteilung keine Arbeitsunfähigkeit vorliege und gegenteiligen Einschätzungen kein Beweiswert zukomme (E. 4.5.3).

\section{Kommentar}

5 Die Sonderbehandlung von depressiven Erkrankungen konnte nach der fundierten Kritik an der Rechtsprechung des Bundesgerichts ${ }^{1}$ nicht von Dauer sein. Das relativ rasche Einlenken des Bundesgerichts ist daher im Ergebnis zu begrüssen. Es erstaunt allerdings, wie wenig eingehend das Gericht seine Praxisänderung begründet hat.

6 Nachdem für die schwer beweisbaren psychosomatischen Krankheiten 2015 die sogenannte Überwindbarkeitspraxis erfreulicherweise aufgegeben und einer differenzierteren Einzelfallbetrachtung zugeführt worden war, konnte sich die neue Praxis nicht halten, allein bei leicht- und mittelgradigen depressiven Störungen entgegen dem Gesetz Therapieresistenz als zusätzliche Anspruchsvoraussetzung $\mathrm{zu}$ verlangen. Im Spannungsfeld von Rechtsgleichheit und Einzelfallbeurteilung hat das Bundesgericht nun zu einem

\footnotetext{
Insbesondere Roman Schleifer, u.a. Der Begriff der Therapieresistenz bei unipolaren depressiven Störungen aus medizinischer und aus rechtlicher Sicht - eine Standortbestimmung im Nachgang zu BGE 9C_13/2016, in: HAVE 3/2017, S. 266274 und Eva Slavik, Invalidenrentenanspruch bei depressiven Erkrankungen; eine kritische Auseinandersetzung mit der Rechtsprechung des Bundesgerichts, in: Jusletter vom 4. September 2017.
} 
Weg (zurück) gefunden, der die medizinischen Erkenntnisse über Depressionen nicht länger ignoriert (E. 4.3) und die rechtlichen Rahmenbedingungen respektiert (E. 4.4). Gesetzliche Voraussetzungen für eine Invalidenrente sind nämlich nicht Therapieresistenz, sondern gesundheitliche Beeinträchtigungen von einer gewissen Schwere und Dauer, welche zu einer Einbusse der Erwerbsmöglichkeiten führen. Dabei ist zu berücksichtigen, ob die betroffene Person im Rahmen der Schadenminderungspflicht alles Zumutbare unternommen hat. $\mathrm{Zu}$ diesen Themen müssen Beweise erhoben, und die Einbusse unter Berücksichtigung der konkreten Berufsmöglichkeiten für die betroffene Person ermittelt werden.

7 Zwei Bemerkungen des Bundesgerichts lassen allerdings aufhorchen und geben Anlass zu einer kritischen Beobachtung der künftigen Rechtsprechung:

Nicht im Urteil selber, aber in der Medienmitteilung dazu schreibt das Bundesgericht, es sei die entscheidende Frage, ob es der betroffenen Person gelinge, den Beweis für eine invalidisierende Einschränkung der Arbeitsfähigkeit zu erbringen $^{2}$. Dies ist insofern zu präzisieren, als es nicht Sache der Versicherten ist, Beweis zu führen, wenngleich sie letztlich die Beweislast tragen. Der Sachverhalt ist vielmehr gem. Art. 43 Abs. 1 ATSG von Amtes wegen festzustellen (Untersuchungsgrundsatz), indem die IV-Stellen die notwendigen Abklärungen vornehmen und die erforderlichen Auskünfte einholen. Anschliessend sind die Beweise zu würdigen. Lässt sich der rechtserheb-

$2 \overline{\text { Medienmitteilung des Bundesgerichts vom 14. De- }}$ zember 2017 (IV-Rente bei psychischen Leiden: Änderung der Rechtsprechung). liche Sachverhalt nicht mit dem hier gültigen Beweismass der überwiegenden Wahrscheinlichkeit erstellen, muss nach der Beweislastregel von Art. 8 ZGB der Antrag auf eine Invalidenrente abgewiesen werden. Je aktiver und gründlicher aber der Sachverhalt zuvor von einem Versicherungsträger abgeklärt wurde, desto bessere Entscheidgrundlagen stehen für eine letztlich gerichtliche Beweiswürdigung zur Verfügung. In der Pflicht sind diesbezüglich also die IVStellen, nicht die Versicherten.

9 In Erwägung 4.5.3 des Urteiles steht ausserdem, aus «Gründen der Verhältnismässigkeit» könne vom strukturierten Beweisverfahren abgewichen und auf die vorhandene Aktenlage abgestellt werden. Sollten damit allfällige Befürchtungen der Politik im Hinblick auf finanzielle Auswirkungen der Praxisänderung zerstreut werden? Laut Art. 33 VwVG (i.V. m. Art. 43 ATSG) nimmt die Behörde die Beweise $a b$, wenn diese zur Abklärung des Sachverhaltes tauglich erscheinen. Der Massstab für den Umfang der Sachverhaltsabklärungen ist demnach die Tauglichkeit weiterer Beweismittel. Nur diesbezüglich besteht ein Ermessensspielraum ${ }^{3}$. Behörden und Gerichte haben also bereits die Möglichkeit, eine antizipierte Beweiswürdigung vorzunehmen, ohne dafür den Grundsatz der Verhältnismässigkeit bemühen zu müssen. Zudem ist das Verhältnismässigkeitsprinzip ursprünglich zum Schutz der Rechtsunterworfenen und nicht zur Durchsetzung der Interessen von Behörden entwickelt worden. Eine Sonderbehandlung bei der Sachverhaltsfeststel-

3 Bernhard Waldmann/Jürg Bickel, Praxiskommentar Verwaltungsverfahrensgesetz (VwVG), 2. Aufl., Zürich 2016, N21 ff. zu Art. 33 VwVG. 
lung im Zusammenhang mit gewissen Krankheitsbildern wäre also nicht zu rechtfertigen.

10 Zudem wird abzuwarten sein, wie das Bundesgericht das strukturierte Beweisverfahren bei den depressiven Störungen umsetzen wird, und ob es dem final ausgestalteten IVG gerecht wird, somatisch und psychisch bedingte Invalidität nicht unterschiedlich zu behandeln. 